\title{
THE BOUNDARY SPECTRUM OF LINEAR OPERATORS IN FINITE-DIMENSIONAL SPACES
}

\author{
YURI LYUBICH \\ Department of Mathematics, Technion \\ Haifa 32000, Israel \\ E-mail: lyubich@techunix.technion.ac.il
}

1. The basic concepts. Let $X$ be an $n$-dimensional real or complex vector space, $n<\infty$. Let $A: X \rightarrow X$ be a linear operator. It is called power bounded if the semigroup $\Pi(A)=\left\{A^{k}\right\}_{0}^{\infty}$ of its natural powers is bounded. (This property can be defined in terms of any norm: $\sup \left\{\left\|A^{k}\right\|: k \in \mathbb{N}\right\}<\infty$. The choice of the norm does not matter.) An operator $A$ is called double power bounded if $A$ is invertible and $A, A^{-1}$ are both power bounded. (Thus, $\sup \left\{\left\|A^{k}\right\|: k \in \mathbb{Z}\right\}<\infty$.) We only consider the power bounded operators. Since in this case the spectral radius $r(A)$ does not exceed 1 , the spectrum $\sigma(A)$ consists of two parts, the boundary (or peripheral) spectrum

$$
\sigma_{1}(A)=\{\lambda: \lambda \in \sigma(A),|\lambda|=1\}
$$

and the interior spectrum

$$
\sigma_{0}(A)=\{\lambda: \lambda \in \sigma(A),|\lambda|<1\} .
$$

(Speaking about spectra in the real case we implicitly change $A$ for its natural complex extension $A_{\mathbb{C}}$.) One of these parts of $\sigma(A)$ may be empty; $\sigma_{1}(A) \neq \emptyset$ iff $r(A)=1$, $\sigma_{0}(A)=\emptyset$ iff $A$ is double power bounded (being power bounded). According to this partition of the spectrum we have the decomposition of the space $X$ into the direct sum

$$
X=X_{1} \dot{+} X_{0}
$$

where $X_{1}$ and $X_{0}$ are invariant subspaces such that

$$
\sigma\left(A \mid X_{1}\right)=\sigma_{1}(A), \quad \sigma\left(A \mid X_{0}\right)=\sigma_{0}(A),
$$

the so-called boundary and interior subspaces. This terminology also applies to the parts of the operator $A, A_{1}=A \mid X_{1}$ and $A_{0}=A \mid X_{0}$. The following quite elementary proposition is in fact the starting point of a rather deep theory presented below.

1991 Mathematics Subject Classification: Primary 47A10.

The paper is in final form and no version of it will be published elsewhere. 
Proposition 1.1. The boundary part $A_{1}$ is double power bounded. The interior part $A_{0}$ is such that $\lim _{k \rightarrow \infty} A_{0}^{k}=0$.

To explain a key role of this statement let us note that $\Pi(A)$ is precompact being a bounded subset of $L(X)$ where $L(X)$ is the space of all linear operators in $X, \operatorname{dim} L(X)=$ $n^{2}<\infty$. The closure $\overline{\Pi(A)}$ is a compact semigroup. In this sense $A$ is an almost periodic operator. It is a finite-dimensional version of a general statement regarding operators in a Banach space or elements of a Banach algebra (see for example [11, Chapter 4]). Proposition 1.1 corresponds to the so-called de Leeuw-Glicksberg decomposition [6] or, in terms of [11], the boundary spectrum splitting-off theorem. There are a lot of interesting applications of the general theory of almost periodic operators to Markov chains (e.g. [3], [10], [19]), dynamical systems (e.g. [7], [8], [12], [13], [16], [22]), harmonic analysis and spectral theory (e.g. [6], [9], [11], [21]). Though this development is rather recent, its roots are in an abstract theory of compact semigroups which arose much earlier. A principal concept in this way is the Sushkevich kernel (S.k.).

2. The Sushkevich kernel. This object can be formally defined as the smallest two-sided ideal of a given semigroup but this may not exist if the semigroup is taken arbitrarily. For example, there is no S.k. in the additive semigroup $\mathbb{N}$. However, the S.k. does exist in any finite semigroup [20] and even in any compact semigroup [17]. Moreover, it has a lot of remarkable properties, among them:

THEOREM 2.1. For any compact commutative semigroup its S.k. is a compact group (whose unit is an idempotent).

Coming back to our case of a power bounded linear operator $A$ in a space $X, \operatorname{dim} X=$ $n<\infty$, we can introduce the following

Definition 2.2. The S.k. of the semigroup $\overline{\Pi(A)}$ is called the S.k. of $A$.

We denote it by $K(A)$. Let us emphasize that $\overline{\Pi(A)}$ is, obviously, commutative, so Theorem 2.1 is applicable. However, we do not need it because the S.k. $K(A)$ can be constructed directly in our context (cf. [4]). For this purpose we consider the projection $P$ connected with the decomposition $(*)$. We call it the boundary projection of the operator $A$ and prove

Lemma 2.3. $P \in \overline{\Pi(A)}$.

Proof. Let $\left\{k_{m}\right\}_{0}^{\infty} \subset \mathbb{N}$ be such that $k_{m+1}-k_{m} \rightarrow \infty$ and the limit $U=\lim _{m \rightarrow \infty} A_{1}^{k_{m}}$ exists. Then

$$
U^{-1}=\lim _{m \rightarrow \infty} A_{1}^{-k_{m}} \quad \text { and } \quad \lim _{m \rightarrow \infty} A_{1}^{k_{m+1}-k_{m}}=U U^{-1}=\mathrm{id}=P \mid X_{1} .
$$

On the other hand, $\lim A_{0}^{k_{m+1}-k_{m}}=0=P \mid X_{0}$. Thus,

$$
P=\lim _{m \rightarrow \infty} A^{k_{m+1}-k_{m}}
$$

Lemma 2.4. For any operator $B \in \overline{\Pi(A)}$ the smallest ideal containing $B$ is closed. Furthermore, $B \overline{\Pi(A)}=\overline{B \Pi(A)}$. 
Proof. This ideal is $B \overline{\Pi(A)}$. It is closed since $\overline{\Pi(A)}$ is compact. Obviously, $\overline{B \Pi(A)} \subset B \overline{\Pi(A)}$. On the other hand, if $C \in B \overline{\Pi(A)}$ then

$$
C=B\left(\lim _{m \rightarrow \infty} A^{q_{m}}\right)=\lim _{m \rightarrow \infty}\left(B A^{q_{m}}\right)
$$

for a sequence $\left\{q_{m}\right\}_{0}^{\infty}$. Passing to a convergent subsequence we obtain $C \in B \overline{\Pi(A)}$.

Corollary 2.5. Every ideal of the semigroup $\overline{\Pi(A)}$ contains a closed ideal.

Now we can get $K(A)$ in the following way.

THEOREM 2.6. The ideal of the semigroup $\overline{\Pi(A)}$ generated by the boundary projection $P$ is its S.k.

$$
K(A)=P \overline{\Pi(A)}
$$

It is a compact group with the unit $P$.

Pr o of. For every ideal $I \subset \overline{\Pi(A)}$ we have to prove that $P \overline{\Pi(A)} \subset I$. One can assume $I$ to be closed because of Corollary 2.5. Let $B \in I$. Then $B$ is a power bounded operator with the same decomposition $(*)$. By Lemma 2.3, $P \in \overline{\Pi(B)} \subset I$. Hence $P \overline{\Pi(A)} \subset I$. Thus, $P \overline{\Pi(A)}$ is the S.k. of $\overline{\Pi(A)}, K(A)=P \overline{\Pi(A)}$.

By Lemma 2.4, $K(A)$ is closed. It is a semigroup being an ideal. The boundary projection $P$ is its unit since $P=P^{2} \in P \overline{\Pi(A)}$ by Lemma 2.3 , and if $B \in K(A)$ then $B=P C$ with $C \in \overline{\Pi(A)}$, hence $P B=P C=B$. Let

$$
B=P\left(\lim _{m \rightarrow \infty} A^{l_{m}}\right)
$$

for a sequence $\left\{l_{m}\right\}_{0}^{\infty}$. Coming back to $(* *)$ one can assume that $k_{m+1}-k_{m}-l_{m} \rightarrow \infty$ and the limit

$$
\widehat{B}=\lim _{m \rightarrow \infty} A^{k_{m+1}-k_{m}-l_{m}}
$$

exists. Then $B \widehat{B}=P^{2}=P$. Thus, $K(A)$ is a group.

Corollary 2.7. If $A$ is double power bounded then $K(A)=\overline{\Pi(A)}$.

We have described $K(A)$ algebraically. The next result yields a dynamical description.

THEOREM 2.8. The S.k. $K(A)$ coincides with the $\Omega$-limit set $\Omega(A)$ of the semigroup $\Pi(A)$.

Proof. First, $K(A) \subset \Omega(A)$ since $\Omega(A)$ is an ideal in $\bar{\Pi}(A)$. Indeed, $\Omega(A)$ is nonempty and $\Omega(A) A^{k} \subset \Omega(A)$ for all $k \in \mathbb{N}$. Since $\Omega(A)$ is closed, we get $\Omega(A) \overline{\Pi(A)} \subset$ $\Omega(A)$. To establish the converse inclusion $\Omega(A) \subset K(A)$ we take $B \in \Omega(A)$, i.e.

$$
B=\lim _{m \rightarrow \infty} A^{l_{m}}
$$

with a sequence $\left\{l_{m}\right\}_{0}^{\infty}, l_{m} \rightarrow \infty$. Then $B \mid X_{0}=0$ hence, $B=P B \in P \overline{\Pi(A)}=K(A)$.

The intrinsic structure of the group $K(A)$ is completely determined by the boundary spectrum $\sigma_{1}(A)$. Let $\sigma_{1}(A)=\left\{\lambda_{1}, \ldots, \lambda_{s}\right\}$. Since all the eigenvalues lie on the unit circle $\mathbb{T}$, one can consider the ordered set $\sigma_{1}(A)$ as a point $a_{1}$ on the $s$-dimensional torus $\mathbb{T}^{s}$ which is a compact group (due to the standard group structure on $\mathbb{T}$ ). 
TheOrem 2.9 ([4], Ch. 1, Th. 2.4). There exists a unique continuous monomorphism $h: K(A) \rightarrow \mathbb{T}^{s}$ such that $h\left(A_{1}\right)=a_{1}$ where $A_{1}$ is the boundary part of $A$.

Proof. Let

$$
A_{1}=\sum_{j=1}^{s} \lambda_{j} P_{j}
$$

be the spectral decomposition, so $P_{j}$ are projections in $X_{1}$ whose images are the corresponding eigenspaces. Moreover,

$$
\sum_{j=1}^{s} P_{j}=\mathrm{id}, \quad P_{j_{1}} P_{j_{2}}=0 \quad\left(j_{1} \neq j_{2}\right) .
$$

Then

$$
A_{1}^{l}=\sum_{j=1}^{s} \lambda_{j}^{l} P_{j}, \quad l \in \mathbb{N} .
$$

If now $B \in K(A)$ then by Theorem 2.8 ,

$$
B=\lim _{m \rightarrow \infty} A^{l_{m}}=\lim _{m \rightarrow \infty} A_{1}^{l_{m}} P
$$

for a sequence $\left\{l_{m}\right\}_{0}^{\infty}, l_{m} \rightarrow \infty$. Hence,

$$
B=\sum_{j=1}^{s} \lambda_{j}(B) P_{j} P \quad \text { where } \quad \lambda_{j}(B)=\lim _{m \rightarrow \infty} \lambda_{j}^{l_{m}}, 1 \leq j \leq s .
$$

(These limits do not depend on the choice of the sequence because $\lambda_{j}(B)$ is the unique eigenvalue of $B$ in the subspace $\operatorname{Im} P_{j}$.) Letting $h(B)=\left(\lambda_{1}(B), \ldots, \lambda_{s}(B)\right)$ we obtain the desired homomorphism. The uniqueness statement is obvious.

COROLlary 2.10. The S.k. $K(A)$ is topologically isomorphic to a closed subgroup of the torus $\mathbb{T}^{s}$ where $s=\operatorname{card}\left[\sigma_{1}(A)\right]$. This subgroup coincides with the semigroup of $\mathbb{T}^{s}$ topologically generated by the point $a_{1}=\sigma_{1}(A)$.

We denote this subgroup by $\left\langle a_{1}\right\rangle$. Every closed subgroup $G \subset \mathbb{T}^{s}$ is of the form $\mathbb{T}^{\rho} \times F$ where $0 \leq \rho \leq s$ and $F$ is a finite group. Indeed, the dual group $G^{*}$ is isomorphic to a factor group of $\left(\mathbb{T}^{s}\right)^{*} \approx \mathbb{Z}^{s}$ so $G^{*}$ is a commutative group generated by some $s$ elements. Therefore $G^{*} \approx \mathbb{Z}^{\rho} \times F$, thus $G \approx G^{* *} \approx \mathbb{T}^{\rho} \times F$.

If $G$ is monothetic then $F$ is cyclic. In particular, we have this information about $K(A)$ in virtue of Corollary 2.10. Now we can describe the parameters $\rho$ and $\operatorname{ord}(F)$ in arithmetical terms concerning the boundary spectrum $\sigma_{1}(A)$.

Let $\lambda_{j}=\exp \left(2 \pi i \theta_{j}\right), 0 \leq \theta_{j}<2 \pi, 1 \leq j \leq s$. These numbers can be treated as vectors from the space $\mathbb{R}$ over the rational field $\mathbb{Q}$. Let

$$
\rho=\operatorname{rank}_{\mathbb{Q}}\left\{\theta_{0}, \theta_{1}, \ldots, \theta_{s}\right\}-1,
$$

where $\theta_{0}=1$. Let $\left\{\theta_{0}, \ldots, \theta_{\rho}\right\}$ be a maximal linearly independent subsystem of the system $\left\{\theta_{0}, \theta_{1}, \ldots, \theta_{s}\right\}$. Then for every $\theta_{j}, j>\rho$, there exists an integer $m_{j} \geq 1$ such that $m_{j} \theta_{j}$ is a linear combination of $\theta_{0}, \ldots, \theta_{\rho}$ with some integer coefficients. Let $m_{j}$ be minimal possible and let $m$ be the least common multiple of $m_{\rho+1}, \ldots, m_{s}$. (In the case $\rho=s$ we set $m=1$.) 
TheOREM 2.11. The S.k. $K(A)$ is topologically isomorphic to $\mathbb{T}^{\rho} \times F$ where $F$ is a cyclic group whose order is a divisor of $m$.

Proof. The point $a_{1}=\left(\lambda_{1}, \ldots, \lambda_{\rho}, \ldots, \lambda_{s}\right) \in \mathbb{T}^{s}$ satisfies some conditions

$$
\lambda_{j}^{m_{j}}=\prod_{q=1}^{\rho} \lambda_{q}^{\omega_{q} j}, \quad \rho+1 \leq j \leq s,
$$

with integer $\omega_{q} j$. The same conditions hold for all points $b \in\left\langle a_{1}\right\rangle$. Consider the canonical projection $r: \mathbb{T}^{s} \rightarrow \mathbb{T}^{\rho}$ keeping the coordinates with numbers $1, \ldots, \rho$; we get $\mathbb{T}^{\rho}=$ $\operatorname{Im}\left(r \mid\left\langle a_{1}\right\rangle\right)$ by the well-known Kronecker theorem. On the other hand, all points $b=$ $\left(\beta_{1}, \ldots, \beta_{\rho}, \ldots, \beta_{s}\right)$ from $\Gamma=\operatorname{ker}\left(r \mid\left\langle a_{1}\right\rangle\right)$ satisfy the conditions

$$
\beta_{1}=\ldots=\beta_{\rho}=1, \quad \beta_{j}^{m_{j}}=1 \quad(\rho+1 \leq j \leq s) .
$$

Therefore $\Gamma$ is finite and $b^{m}=e\left(e\right.$ is the unit of $\left.\mathbb{T}^{s}\right)$ for all $b \in \Gamma$.

We have $\left\langle a_{1}\right\rangle / \Gamma \approx \mathbb{T}^{\rho}$ and we know that $\left\langle a_{1}\right\rangle$ is a direct product of a torus and a cyclic group $F$. Then this torus must be $\mathbb{T}^{\rho}$ (up to topological isomorphism) and $F \approx \Gamma$, so $\operatorname{ord}(F)$ is a divisor of $m$.

The question about the exact value of $\operatorname{ord}(F)$ remains open.

COROLlary 2.12. $K(A)$ is infinite if and only if this group contains a subgroup which is topologically isomorphic to $\mathbb{T}$.

It is just the case $\rho \geq 1$. The opposite case $\rho=0$ is such that all $\theta_{j}, 1 \leq j \leq s$, are rational or, equivalently, all $\lambda_{j}, 1 \leq j \leq s$, are roots of 1 . Then we say that the boundary spectrum is rational.

Corollary 2.13. $K(A)$ is finite if and only if the boundary spectrum $\sigma_{1}(A)$ is rational.

This property is of special interest from the dynamical point of view because every trajectory $\left\{A^{k} x\right\}_{k=0}^{\infty}, x \in X$, converges to a limit cycle iff $K(A)$ is finite. By Corollary 2.13 we have a spectral criterion of the cyclic limit behavior: the rationality of the boundary spectrum

Note that if $\sigma_{1}(A)$ is rational, so $\lambda_{j}^{m_{j}}=1,1 \leq j \leq s$, and $m_{j}$ are minimal possible as before then $K(A)$ is a cyclic group of order $\vartheta=$ the least common multiple of $m_{j}$. This $m$ is the length of the limit cycles of the trajectories $\left\{A^{k} x\right\}_{k=0}^{\infty}$ for almost all $x$.

Below we investigate the problem of rationality of the boundary spectrum relating to the space $X$ endowed with an additional geometric structure.

3. Criteria of the rationality of the boundary spectrum. From now on we only consider the real space $X$. As a simplest example let us recall the stochastic operators in $\mathbb{R}^{n}$. The rationality of boundary spectrum in this case was established already by Frobenius. There are at least two independent geometrical reasons for that property, namely, any stochastic operator $A$ in $\mathbb{R}^{n}$ is: 1) a contraction with respect to the coordinate sup-norm and 2) monotone with respect to the coordinate ordering. Both of them can be included in a more general context. 
Let us define a convex structure in $X$ as a pair $(X, D)$ where $D \subset X$ is a convex closed body such that $0 \in D$. (The term "body" means that $\operatorname{Int} D \neq \emptyset$.)

A linear operator $T: X \rightarrow X$ such that $T D \subset D$ is called an endomorphism of the convex structure (or D-endomorphism). If, moreover, $T$ is invertible and $T^{-1}$ is also an $D$-endomorphism then $T$ is called a $D$-automorphism. The set $\operatorname{End}(X, D)$ of all $D$ endomorphisms is a semigroup with the usual multiplication of operators. The identity operator $I$ is the unit of this semigroup. The set $\operatorname{Aut}(X, D)$ of all D-automorphisms is just the multiplicative group of the semigroup $\operatorname{End}(X, D)$. Note that $\operatorname{Aut}(X, D) \neq \operatorname{End}(X, D)$ since the zero operator belongs to $\operatorname{End}(X, D)$.

A convex structure $(X, D)$ is called symmetric if $-D=D$. It is called completely nonsymmetric if $(-D) \cap D=\{0\}$.

ExAmple 3.1. Let $X$ be a normed space and let $D$ be the unit ball, $D=\{x:\|x\| \leq 1\}$. Then $(X, D)$ is a symmetric convex structure. Its endomprhisms are just contractions, $\|A\| \leq 1$, and its automorphisms are just isometries. An additional property of $D$ in this example is compactness. Note that $\operatorname{End}(X, D)$ and $\operatorname{Aut}(X, D)$ are also compact in this case.

ExAmple 3.2. Let $X$ be an ordered space and let $D$ be the nonnegative cone, $D=\{x: \quad x \geq 0\}$. If $D$ is solid, i.e. $\operatorname{Int} D \neq \emptyset$, then $(X, D)$ is a convex structure but it is completely nonsymmmetric. The semigroup $\operatorname{End}(X, D)$ consists of all monotone operators. It is noncompact because all homotheties $\lambda I, \lambda>0$, are $D$-endomorphisms. They are even $D$-automorphisms, so $\operatorname{Aut}(X, D)$ is also noncompact. This is nonclosed in $\operatorname{End}(X, D)$ since $\lambda I \rightarrow 0$ as $\lambda \rightarrow 0$ but $\operatorname{End}(X, D)$ is obviously closed. As usual, $D$-endomorphisms are also called the nonnegative operators in this case.

We say that a subspace $Y \neq 0$ is admissible if $D_{Y}=D \cap Y$ is a body in $Y$. In this case we can define a convex structure $\left(Y, D_{Y}\right)$ called a substructure of $(X, D)$. Note that if $0 \in \operatorname{Int} D$ then all subspaces $Y \neq 0$ are admissible. We call a subspace $Y \neq 0$ $D$-complemented if there exists a projection $Q \in \operatorname{End}(X, D) \operatorname{such}$ that $\operatorname{Im} Q=Y$. In the case of the unit ball $D$ in a normed space $X$ that means $\|Q\|=1$ and $\operatorname{Im} Q=Y$; $Q$ is called an orthoprojection onto $Y$ and $Y$ is orthogonally complemented if such a $Q$ does exist. In the case of a cone $D$, we say a positively complemented subspace for a D-complemented one.

Lemma 3.3. Every D-complemented subspace is admissible.

Proof. If $Y=\operatorname{Im} Q$ where $Q \in \operatorname{End}(X, D)$ is a projection, then $D_{Y}=Q D$. Hence, $\operatorname{Int}_{Y} D_{Y} \neq \emptyset$ since any projection is an open mapping onto its image.

We are especially interested in power bounded $D$-endomorphisms. They form a semigroup denoted by $\operatorname{End}_{\infty}(X, D)$. Accordingly, $\operatorname{Aut}_{\infty}(X, D)$ is a group of double power bounded $D$-automorphisms.

Lemma 3.4. Let $A \in \operatorname{End}_{\infty}(X, D)$ and let $P$ be the boundary projection of $A$. Then $P \in \operatorname{End}(X, D)$.

Proof. $\overline{\Pi(A)} \subset \operatorname{End}(X, D)$ and $P \in \overline{\Pi(A)}$ by Lemma 2.3 . 
Corollary 3.5. If $A \in \operatorname{End}_{\infty}(X, D)$ then its boundary subspace $X_{1}$ is D-complemented.

By Lemma 3.3 it is admissible. Now we consider the boundary part $A_{1}=A \mid X_{1}$ in the substructure $\left(X_{1}, D_{1}\right)$, where $D_{1}=D \cap X_{1}$.

Lemma 3.6. If $A \in \operatorname{End}_{\infty}(X, D)$ then $K\left(A_{1}\right) \subset \operatorname{Aut}_{\infty}\left(X_{1}, D_{1}\right)$. In particular, $A_{1} \in$ $\operatorname{Aut}\left(X_{1}, D_{1}\right)$.

Proof. First, $A_{1}$ is a double power bounded operator. Obviously, $\overline{\Pi\left(A_{1}\right)} \subset$ $\operatorname{End}\left(X_{1}, D_{1}\right)$. By Corollary 2.7, $K\left(A_{1}\right) \subset \operatorname{End}\left(X_{1}, D_{1}\right)$.

Everything is prepared to prove the following basic result.

TheOREM 3.7. For a convex structure $(X, D)$ the following properties are equivalent.

1) The boundary spectrum of every power bounded D-endomorphism is rational.

2) For every $D$-complemented subspace $Y$ the group of $D_{Y}$-automorphisms does not contain any infinite compact subgroup.

3) For every D-complemented subspace $Y$ the group of $D_{Y}$-automorphisms does not contain a subgroup which is topologically isomorphic to $\mathbb{T}$.

Pro of. 1$) \Rightarrow 2)$. Let $\Gamma \subset \operatorname{Aut}\left(Y, D_{Y}\right)$ be a compact subgroup and let $Q$ be a projection onto $Y, Q \in \operatorname{Aut}\left(X, D_{X}\right)$. We consider the subset of $\mathbb{T}$ defined as

$$
\sigma(\Gamma)=\bigcup_{V \in \Gamma} \sigma(V)
$$

First of all, this "united spectrum" is rational because for every $V \in \Gamma$ we have $V Q \in$ $\operatorname{End}_{\infty}(X, D)$ and $\sigma(V)=\sigma_{1}(V Q)$.

Secondly, $\lambda \in \sigma(\Gamma) \Rightarrow \lambda^{k} \in \sigma(\Gamma)$ for all integers $k$ because of $V \in \Gamma \Rightarrow V^{k} \in \Gamma$. Finally, the set $\sigma(\Gamma)$ is closed because $\Gamma$ is compact.

With these properties the subset $\sigma(\Gamma) \subset \mathbb{T}$ must be finite. Hence, there exists an integer $q \geq 1$ such that $\lambda^{q}=1$ for all $\lambda \in \sigma(\Gamma)$. Then $V^{q}=$ id for all $V \in \Gamma$. Letting $R=V-$ id we obtain

$$
\sum_{j=1}^{q}\left(\begin{array}{l}
q \\
j
\end{array}\right) R^{j}=0
$$

which implies $R=0$ if $R$ is small enough. Thus, the unit element is isolated in $\Gamma$, which means that $\Gamma$ is discrete. Being compact, the group $\Gamma$ is finite.

$2) \Rightarrow 3$ ) trivially.

$3) \Rightarrow 1)$. Let $A \in \operatorname{End}_{\infty}(X, D)$ and let $X_{1}$ be its boundary subspace, $D_{1}=D \cap X_{1}$ and $A_{1}=A \mid X_{1}$ as usual. By Corollary 3.5, $X_{1}$ is $D$-complemented. By Lemma 3.6 and Corollary 2.12 condition 3 ) implies that $K\left(A_{1}\right)$ is finite. Therefore $\sigma\left(A_{1}\right)=\sigma_{1}(A)$ is rational.

For the unit balls the equivalence 1$) \Leftrightarrow 2$ ) was obtained in [9] in the same way as above. For the cones it was done in [21]. In the first of those cases the group $\operatorname{Aut}\left(Y, D_{Y}\right)$ is automatically compact, so 2) only means that this group is finite as stated in [9]. However, the main results of those papers are some purely geometric criteria of rationality of the boundary spectrum. 
TheOREm 3.8 [9]. Let $X$ be a normed space. Then the boundary spectrum of every contraction in $X$ is rational if and only if there is no orthogonally complemented 2-dimensional subspace $Y \subset X$ such that the disk $D_{Y}$ is Euclidean.

The last property means that there exists an inner product (, ) in $Y$ such that

$$
D_{Y}=\{y: y \in Y,(y, y) \leq 1\} .
$$

One can say that $Y$ is a Euclidean plane.

COROllary 3.9 [9]. The boundary spectrum of every contraction in $l_{p}^{n}, 1 \leq p \leq \infty$, $p \neq 2$ is rational.

The point is that there are no Euclidean planes in $l_{p}^{n}$ except for $p$ which are even integers. In the last case a Euclidean plane may exist. This depends on $n$, the criterion of existence is $n \geq p / 2[15,18]$, so the simplest example is $l_{4}^{3}$ (given in [9]). However, there are no orthogonally complemented Euclidean planes in $l_{p}^{n}$ for any $p \neq 2$ and an $n[9]$.

As a sufficient condition the absence of Euclidean planes was first established in [5]. This yields Corollary 3.9 except for $p=4,6, \ldots$ Moreover, this also provides

Corollary 3.10. If $X$ is a polyhedral normed space then the boundary spectrum of every contraction in $X$ is rational.

A similar theory can be developed for cones [1], [5], [21]. A final result is the following.

THEOREM 3.11 [21]. Let $X$ be ordered by a solid cone D. Then the boundary spectrum of every power bounded nonnegative operator in $X$ is rational if and only if there is no positively complemented 3-dimensional subspace $Y$ such that the cone $D_{Y}$ is Euclidean.

The last property means that there exists a direct decomposition

$$
Y=Y_{1} \dot{+} Y_{2}, \quad \operatorname{dim} Y_{1}=1, \operatorname{dim} Y_{2}=2,
$$

such that

$$
D_{Y}=\left\{y: y=y_{1}+y_{2}, y_{1} \in Y_{1}, y_{2}=Y_{2}, y_{1} \geq \sqrt{\left(y_{2}, y_{2}\right)}\right\}
$$

where $Y_{1}$ is identified with $\mathbb{R}$ and $($,$) is an inner product in Y_{2}$. (Another name for such a cone is "Lorentzian".)

COROllary 3.12. Let $X$ be ordered by a solid polyhedral cone. Then the boundary spectrum of every power bounded nonnegative operator in $X$ is rational.

The Euclidean cone construction can be generalized in the following way (cf. [2], [14]). Let $Z$ be normed space and let $X=\mathbb{R} \oplus Z$ be the outer direct sum, so

$$
X=\left\{x: x=\left(\begin{array}{l}
\xi \\
z
\end{array}\right), \xi \in \mathbb{R}, z \in Z\right\} .
$$

A natural cone in this 1-dimensional extension can be introduced as

$$
D=\left\{\left(\begin{array}{l}
\xi \\
z
\end{array}\right): \xi \geq\|z\|\right\} .
$$

We call it the hyperbolic cone over the space $Z$. (The reason for this name comes from the Euclidean case, because if $\|z\|^{2}=(z, z)$ then the inequality $\xi \geq\|z\|$ is equivalent to $\xi^{2}-(z, z) \geq 0$ (with the restriction $\xi \geq 0$ ) and this quadratic form is hyperbolic.) 
Corollary 3.13 [21]. Let the space $X=\mathbb{R} \oplus l_{p}^{n-1}, 1 \leq p \leq \infty, p \neq 2$, be provided with the hyperbolic cone. Then the boundary spectrum of every power bounded nonnegative operator in $X$ is rational.

It is interesting to extend the previous theory to general convex structures. At present the author has the following preliminary results.

TheOrem 3.14. Let $(X, D)$ be a convex structure such that $0 \in \operatorname{Int} D$. If there are no 2-dimensional Euclidean substructures of this structure then the boundary spectrum of every power bounded D-endomorphism is rational.

We say that an admissible 3-dimensional subspace $Y$ is semi-Euclidean if $D_{Y}$ is a rotation body which means that $Y=Y_{1}+Y_{2}, \operatorname{dim} Y_{1}=1, \operatorname{dim} Y_{2}=2$ and $D_{Y}$ is the set of $y=y_{1}+y_{2}$ with $y_{1} \in Y_{1}, y_{2} \in Y_{2}$ satisfying the inequality

$$
\sqrt{\left(y_{2}, y_{2}\right)} \leq d\left(y_{1}\right)
$$

where $d$ is a concave nonnegative function on a closed finite or infinite interval of $Y_{1} \equiv \mathbb{R}$. In some extreme cases this function may take the value $+\infty$ which yields the space $Y$ or some of its half-spaces or a layer in $Y$ as extreme examples. If the domain of $d$ is the whole axis $\mathbb{R}$ then $d$ is constant and $D_{Y}$ is a cylinder.

TheOrem 3.15. Let $(X, D)$ be a convex structure such that $0 \in \partial D$. If there is no 3-dimensional semi-Euclidean substructure of this structure then the boundary spectrum of every power bounded D-endomorphism is rational.

In conclusion let us formulate the following

Problem. Let the boundary spectrum of a $D$-endomorphism $A$ be rational. How can one characterize the order of the group $K(A)$ in geometrical terms?

\section{References}

[1] G. P. Barker and R. E. L. Turner, Some observations on the spectra of cone preserving maps, Linear Algebra Appl. 6 (1973), 149-153.

[2] M. Fiedler and E. Haynsworth, Cones which are topheavy with respect to a norm, Linear and Multilinear Algebra 1 (1973), 203-211.

[3] B. Jamison, Asymptotic behavior of successive iterates of continuous functions under a Markov operator, J. Math. Anal. Appl. 9 (1964), 203-214.

[4] M. A. Kaashoek and T. T. West, Locally Compact Semi-Algebras with Applications to Spectral Theory of Positive Operators, North-Holland, Amsterdam, 1974.

[5] M. A. Krasnosel'skiǔ, On a spectral property of linear completely continuous operators in the space of continuous functions, Probl. Mat. Anal. Slozhnykh Sistem 2 (1968), 68-71 (in Russian).

[6] K. de Leeuw and I. Glicksberg, Applications of almost periodic compactifications, Acta Math. 105 (1961), 63-97.

[7] M. Yu. Lyubich, On the maximal entropy measure of a rational endomorphism of the Riemann sphere, Funktsional. Anal. i Prilozhen. 16 (1982), 78-79 (in Russian). 
[8] M. Yu. Lyubich and Yu. I. Lyubich, The Perron-Frobenius theory for almost periodic operators and representations of semigroups, J. Soviet Math. 48:5 (1990), 539-554; transl. from Teor. Funktsiü Funktsional. Anal. i Prilozhen. 46 (1986), 54-72.

[9] Yu. I. Lyubich, On the boundary spectrum of contractions in Minkowski spaces, Siberian Math. J. 11 (2) (1970), 271-279.

[10] - , The iterations of quadratic mappings, in: Mathematical Economics and Functional Analysis, Nauka, Moscow, 1974 (in Russian).

[11] - Introduction to the Theory of Banach Representations of Groups, Birkhäuser, Basel, 1988.

[12] - Dissipative actions and almost periodic representations of Abelian semigroups, Ukrainian Math. J. 40 (1988), 58-62.

[13] - On trajectory convergence of dissipative flows in Banach spaces, Integral Equations Oper. Theory 13 (1990), 138-144.

[14] - , Perron-Frobenius theory for finite-dimensional spaces with a hypebolic cone, Linear Algebra Appl. 220 (1995), 283-309.

[15] Yu. I. Lyubich and L. N. Vaserstein, Isometric embeddings between classical Banach spaces, cubature formulas and spherical designs, Geom. Dedicata 47(1993), 327-362.

[16] Yu. I. Lyubich and Vũ Quôc Phóng, Asymptotic stability of linear differential equations in Banach spaces, Studia Math. 88 (1988), 37-42.

[17] K. Numakura, On bicompact semigroups, Math. J. Okayama Univ. 1(1952), 99-108.

[18] B. Reznick, Sums of even powers of real linear forms, Mem. Amer. Math. Soc. 463 (1992).

[19] M. Rosenblatt, Eigencontinuous Markov operators, Teor. Veroyatnost. i Primenen. 9 (1964), 205-222.

[20] A. K. Suschkevitsch, Über die endlichen Gruppen ohne das Gesetz der eindeutigen Umkehrbarkeit, Math. Ann. 99 (1928), 30-50.

[21] A. I. Veitsblit and Yu. I. Lyubich, Boundary spectrum of nonnegative operators, Siberian Math. J. 26 (1985), 798-802.

[22] Vũ Quôc Phóng, Almost periodic and strongly stable semigroups of operators, this volume, 401-426. 